\title{
The Chemical-Composition Distribution of Styrene-Acrylonitrile Copolymer Synthesized by Radiation-Induced Copolymerization at Low Temperature
}

\author{
Shinya Teramachi* and Hisashi Uchiyama \\ Department of Applied Chemistry, Faculty of Engineering, \\ Nagoya University, Nagoya, Japan.
}

(Received February 19, 1973)

\begin{abstract}
A styrene-acrylonitrile copolymer was synthesized by radiation-induced copolymerization in ethyl bromide at $-78^{\circ} \mathrm{C}$.

The chemical-composition distribution curve of the sample obtained from the results of compositional fractionation is not continuous, but shows two distinctly different peaks. The acrylonitrile contents of the two peaks are close to those of copolymers polymerized by anionic and free-radical mechanisms, respectively. From this result, it becomes clear that the copolymer sample is a mixture of two kinds of copolymers polymerized by both mechanisms, and it is also clear that both anionic and free-radical mechanisms coexist in this copolymerization process.

Moreover, it is shown that the copolymer may have a chemical composition corresponding to an anionic mechanism only if the reaction mixture is highly dried under vacuum.
\end{abstract}

KEY WORDS Radiation-Induced Copolymerization / Styrene-Acrylonitrile Copolymer / Chemical-Composition Distribution / Fractionation /

In polymerizations induced by radiation, the reaction generally proceeds either by a freeradical mechanism or by an ionic mechanism, depending on the experimental conditions. Sometimes however, both free-radical and ionic polymerization may proceed simultaneously. In that case, it has been reported $^{1-6}$ that the monomer-copolymer composition curve is often situated at an intermediate position between the curves related to the free-radical and the ionic mechanisms. It is likely that the relative importance of the contributions from both mechanisms is affected by suppression or acceleration of each mechanism due to the effects of temperature, impurities, additions of solvents or inhibitors, etc.

However, it is not yet clear whether those polymerizations are initiated by two different free-radical and ionic initiators, or by an ion-

* Present address: Department of Industrial Chemistry, Kogakuin University, Nakano-Cho 2665-1, Hachioji, Japan. radical initiator. Tsuda ${ }^{6}$ first tried to solve the problem by fractionating a styrene-acrylonitrile copolymer synthesized by radiation-induced copolymerization. The copolymers initiated by free-radical and by anionic species must have different chemical compositions, as shown later. Hence, it is possible to answer the above question by carefully fractionating the copolymer as function of the chemical composition. From Tsuda's results, it was suggested that the copolymerization is initiated from an anion-radical such as

$$
. \mathrm{CH}=\mathrm{CH}-\mathrm{CH}=\mathrm{N} \ominus
$$

and, consequently, that the copolymer produced is in fact a block copolymer, one block consisting of the anionic copolymer and the other of the free-radical copolymer.

However, considering recent progress in copolymer fractionation as function of chemical composition, it may now be appropriate to reexamine the above conclusion. It is the purpose of the present work to determine whether 


\section{S. Teramachi and H. Uchiyama}

the radiation-induced copolymerization of styrene and acrylonitrile is initiated from two different initiators or from an anion-radical initiator by determining the chemical composition distribution of the copolymer by compositional fractionation.

\section{EXPERIMENTAL}

\section{Polymerization}

Ethyl bromide was used as the solvent. It is known from Tsuda's data ${ }^{6}$ that a copolymer having an intermediate composition between that of the free-radical and the anionic is obtained using ethyl bromide as the solvent. Research grade ethyl bromide was washed with concentrated sulfuric acid and distilled water, dried over calcium chloride and distilled. Styrene and acrylonitrile were washed several times with dilute aqueous solution of sodium hydroxide and distilled water, and then dried over calcium chloride. Just before use, the monomers were distilled under reduced nitrogen atmosphere. In the polymerization of sample $I$, the monomers and the solvent thus purified were sealed into a polymerization ampoule. On the contrary, in the polymerization of sample II, solvent and monomers were mixed together with calcium chloride into the glass bulb attached to the high vacuum system, dried by calcium chloride in the bulb, and then transferred into the polymerization ampoule by vacuum distillation. In this case, therefore, the monomers and the solvent were highly dried. Solutions containing $25 \%$ weight of the monomer mixture with a composition shown in Table I were subject to irradiation by $\gamma$-rays from $\mathrm{Co}^{60}$ source at $-78^{\circ} \mathrm{C}$. The dose rates and the total doses were $1.6 \times$ $10^{5} \mathrm{rad} / \mathrm{hr}$ and $3.6 \times 10^{7} \mathrm{rad}$ for sample $\mathrm{I}$, and $4.0 \times 10^{5} \mathrm{rad} / \mathrm{hr}$ and $1.9 \times 10^{7} \mathrm{rad}$ for sample II, respectively. After the irradiation, the solutions were poured into excess methanol. The co-

Table I.

\begin{tabular}{rccc}
\hline \multirow{2}{*}{ Sample } & \multicolumn{2}{c}{ AN content, mole fract. } & \\
\cline { 2 - 3 } & $\begin{array}{c}\text { Monomer } \\
\text { mixture }\end{array}$ & Copolymer & \\
\hline I & 0.179 & 0.439 & 1.0 \\
II & 0.329 & 0.840 & 0.4 \\
\hline
\end{tabular}

polymers thus precipitated were washed with methanol and dried in vacuo at room temperature. The acrylonitrile contents of the samples were determined by micro-Dumas method of nitrogen analysis.

\section{Fractionation}

In order to carry out chemical-composition fractionation, it is necessary to find a combination of solvent and nonsolvent such that the difference in the interaction parameters with monomer units $A$ and $B$ is as large as possible. The solubility of the copolymer sample I, having an intermediate composition between the freeradical and the anionic ones, was tested in various solvents. The sample was only partially soluble in benzene, toluene, methyl ethyl ketone, chloroform, and others, which are solvents for polystyrene but nonsolvents for polyacrylonitrile, and also in ethylene carbonate and ethylene cyanohydrin, which are solvents for polyacrylonitrile but nonsolvents for polystyrene. All solutions were turbid even when the temperature was raised to $80^{\circ} \mathrm{C}$ or close to the boiling points of those solvents. It was observed that the unsoluble part of the copolymer precipitated at the bottom of the test tube after a day.

The sample was thoroughly soluble in dimethylformamide which is a common solvent for polystyrene and polyacrylonitrile. However, precipitation was not observed when the solvents mentioned above (the solvents for either polystyrene or polyacrylonitrile) were added to dimethylformamide solutions of the sample. From the above results, the conclusion was reached that it is difficult to fractionate the sample by the conventional method, i.e., by varying the mixing ratio of two appropriate solvents. That is, in order to fractionate this sample as a function of the chemical composition, it is necessary to employ various solvents covering a wide range of solvent power. The solvents employed in this work are therefore cyclohexane, toluene, benzene, methyl ethyl ketone and dimethylformamide, and are shown in Table II.

The fractionation was carried out by an ordinary column-elution method. The sample was deposited on glass beads, having a size between 100 and 200 mesh, by evaporating the solvent, dimethylformamide, slowly. The glass beads 


\section{Composition Distribution of Radiation-Polymerized Copolymer}

Table II.

\begin{tabular}{|c|c|c|c|c|}
\hline Fraction no. & $W_{i}, \mathrm{mg}$ & $\omega_{i}$ & AN content, mole fract. & Solvent mixture, volume ratio \\
\hline 1 & 411.9 & 0.4134 & 0.0772 & cyclohexane \\
\hline 2 & 38.8 & 0.0389 & 0.157 & cyclohexane/toluene $(7: 3)$ \\
\hline 3 & 15.6 & & & cyclohexane/toluene $(3: 7)$ \\
\hline 4 & 11.0 & 00557 & 0177 & toluene \\
\hline 5 & 20.0 & 0.0351 & 0.171 & toluene/benzene $(1: 1)$ \\
\hline 6 . & $8.9)$ & & & benzene \\
\hline 7 & 86.2 & 0.0865 & 0.719 & benzene/methyl ethyl ketone $(1: 1)$ \\
\hline 8 & 1.8 & & & methyl ethyl ketone \\
\hline 9 & 8.2 & & & dimethylformamide/benzene $(5: 995)$ \\
\hline 10 & $0.8\}$ & 0.0143 & - & dimethylformamide/benzene $(2: 98)$ \\
\hline 11 & 3.5 & & & dimethylformamide/benzene $(4: 96)$ \\
\hline 12 & 72.8 & 0.0731 & 0.839 & dimethylformamide/benzene $(1: 9)$ \\
\hline 13 & 65.8 & 0.0660 & 0.779 & dimethylformamide/benzene $(3: 7)$ \\
\hline 14 & 49.2 & 0.0494 & 0.852 & dimethylformamide/benzene $(1: 1)$ \\
\hline 15 & 158.9 & 0.1595 & 0.841 & dimethylformamide/benzene $(7: 3)$ \\
\hline 16 & 43.0 & 0.0432 & 0.952 & dimethylformamide \\
\hline
\end{tabular}

coated by the polymer sample were dried out in vacuo for about $50 \mathrm{hr}$ and passed through a sieve of 50 mesh. The copolymer sample charged was $1.1671 \mathrm{~g}$. Each fraction was concentrated by vacuum evaporation, precipitated by pouring into methanol and dried in vacuo for about $24 \mathrm{hr}$. Determination of acrylonitrile content in each fraction was carried out by the same method as in the original sample.

\section{RESULTS}

The compositions of the monomer mixtures used and the acrylonitrile contents of the copolymers obtained as well as conversions are shown in Table I. The relationship between the composition of monomer mixtures used and the composition of the samples obtained is shown in Figure 1 together with the theoretical curves for anionic and free-radical copolymerizations, which were calculated using the reactivity ratios reported in literature..$^{8-10}$ Tsuda's data in ethyl bromide ${ }^{6}$ are also shown in the figure.

The chemical composition of sample II obtained from the highly dried copolymerization system almost agreed with the theoretical value calculated using a pair of reactivity ratios reported for anionic copolymerization by Zutty and Welch. ${ }^{9}$ On the other hand, the composition of sample I obtained from the "wet system"

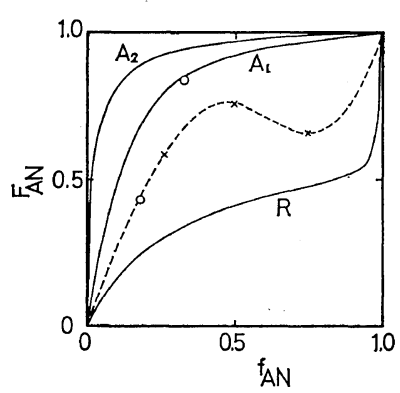

Figure 1. Monomer-copolymer composition curves: $F_{\mathrm{AN}}$, mole fraction of $\mathrm{AN}$ unit in copolymer; $f_{\mathrm{AN}}$, mole fraction of $\mathrm{AN}$ in monomer mixture; $\mathrm{A}_{1}$, calculated curve for anionic copolymerization using $r_{1}(\mathrm{AN})=14$ and $r_{2}(\mathrm{St})=0.20 ; 9 \quad \mathrm{~A}_{2}$, calculated curve for anionic copolymerization using $r_{1}=33$ and $r_{2}=0.005 ; 10 \mathrm{R}$, calculated curve for radical copolymerization using $r_{1}=0.03$ and $r_{2}=0.52 ;^{8}$ $O$, experimental data of the present work; $\times$, Tsuda's data. ${ }^{6}$

does not agree with any theoretical value calculated from the reactivity ratios reported, but is situated on the extrapolation curve from the data of Tsuda. This fact shows that the relative contributions of anionic and free-radical mechanisms to the copolymerization process is affected not only by the solvent species but also by the degree of drying.

Results of the fractionation were shown in Table II. The recovery of the sample was 


\section{S. Teramachi and H. UChiyama}

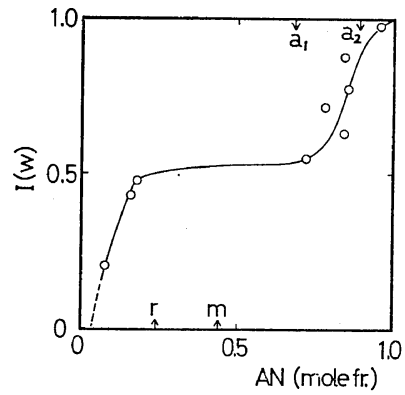

Figure 2. Integral-chemical-composition curve of sample I: $m$, average composition of the sample; $a_{1}$, anionic composition calculated using $r_{1}=14$ and $r_{2}=0.20 ; 9 a_{2}$, anionic composition calculated using $r_{1}=33$ and $r_{2}=0.005 ; 10 r$, radical composition calculated using $r_{1}=0.03$ and $r_{2}=0.52 .8$

$85.4 \%$. The average acrylonitrile content calculated from the fractionation results agreed with that of the original sample with an error of $0.1 \%$.

As shown in Figure 2, the chemical-composition distribution curve of sample I obtained from the fractionation results is not continuous but shows two distinctly different peaks. The acrylonitrile contents of the two peaks are close to those of copolymers polymerized by anionic and free-radical mechanisms, respectively. (The components with higher and lower AN content in sample $\mathrm{I}$ are indicated as $\mathrm{H}$ and $\mathrm{L}$, respectively.)

\section{DISCUSSION}

From the fractionation results shown in Figure 2 , it is clear that the copolymer sample is not a block copolymer as stated by Tsuda, but a mixture of two kinds of copolymers polymerized by anionic and free-radical mechanisms, respectively. If the sample were such a block copolymer as stated by Tsuda, the chemical composition of the sample should be broadly distributed between the anionic and the free-radical compositions.

It was pointed out that the true distribution curve of chemical compositions cannot generally be obtained by one-direction fractionation, i.e., by a fractionation carried out in one fractionation system because of the effect of molecular weight on fractionation. ${ }^{7,11}$ Even when the sample is a mixture of two components differ- ing in chemical composition, therefore, it is possible that the sample is fractionated into a multitude of fractions having continuously variable compositions. This could happen with a system of low fractionation efficiency and Tsuda's results may be due to such an effect.

Thus, it is clear that both anionic and freeradical mechanisms coexist in this copolymerization process. In the radiation-induced copolymerization of styrene and $p$-chlorostyrene at $-40^{\circ} \mathrm{C}$, the coexistence of cationic and freeradical mechanisms was confirmed by using diethylamine and 1,1-diphenyl-2-picryhydrazyl which are inhibitors for the corresponding mechanisms of copolymerization. ${ }^{1}$ The present results agree with the above conclusion in the sense that anionic and free-radical mechanisms coexist, and in addition, it was confirmed that the copolymerization processes are not initiated from an anion-radical. However, the present results do not necessarily deny the existence of the anionradical of acrylonitrile.

The chemical composition of sample II obtained from a highly dried system agrees with the value calculated using a pair of reactivity ratios, $r_{1}=14$ (for acrylonitrile; AN) and $r_{2}=$ 0.20 (for styrene; $\mathrm{St}$ ). ${ }^{9}$ However, this agreement may be rather fortuitous since the values of the reactivity ratios were obtained under quite different experimental conditions, i.e., at $-12^{\circ} \mathrm{C}$ with $n$-buthyl lithium. In fact, the chemical composition of the component $\mathrm{H}$ in sample $\mathrm{I}$, which should be obtained only by an anionic mechanism, does not agree with the value calculated using the above pair of reactivity ratios, but it is close to the value calculated using $r_{1}=33$ and $r_{2}=0.005$ obtained by Mezhirova, Sheinker, and Abkin ${ }^{10}$ as shown in Figure 2. These values of reactivity ratios were obtained by radiation-induced copolymerization at $-78^{\circ} \mathrm{C}$ in dimethyl formamide solution and dried more carefully than in the present experiments. Taking into account the difference between the solvents used by Abkin, et al., and by the present authors, it may be concluded that the reactivity ratios for an anionic copolymerization of styrene and acrylonitrile at $-78^{\circ} \mathrm{C}$ are given by the values of Abkin, et al., and the component $\mathrm{H}$ was obtained by an anionic mechanism only. This may mean that there was some contribution from a radical 
mechanism even in sample II.

Thus, it is also possible that the component $\mathrm{L}$ may be due to a radical mechanism. Moreover, it was shown by Tsuda $^{6}$ that if the reaction mixtures of $\mathrm{St}$ and $\mathrm{AN}$ contain fairly large amounts of water, the chemical compositions of the samples obtained by radiation-induced polymerization are situated in the vicinity of the curve calculated using the reactivity ratios for the radical mechanism. Considering that anionic polymerization is almost stopped by the presence of water, the polymerization in this wet mixture may be mainly by a radical mechanism.

In fact, the component $\mathrm{L}$ has the chemical composition close to the value calculated using $r_{1}=0.03$ and $r_{2}=0.52$ reported for a radical copolymerization. ${ }^{8}$ Therefore, it may be concluded that the component $\mathrm{L}$ in sample $\mathrm{I}$ is synthesized by a radical mechanism only.

Unfortunately, the reactivity ratios for radical copolymerizations of St and AN were all obtained at 40 to $80^{\circ} \mathrm{C}$. Strictly speaking, it is meaningless to compare the present data obtained at $-78^{\circ} \mathrm{C}$ with those values. Nevertheless, it is interesting to see that the chemical composition calculated using the values of $r_{1}$ and $r_{2}$ obtained at higher temperatures are situated in the vicinity of the composition of the component $\mathrm{L}$. This may mean that the temperature dependence of $r_{1}$ and $r_{2}$ in the radical copolymerization of $\mathrm{St}$ and AN may not be so high as reported by Goldfinger and Steidlitz. ${ }^{13}$ If one employs the activation energies reported by them to estimate $r_{1}$ and $r_{2}$ at $-78^{\circ} \mathrm{C}$, the AN content calculated using values of $r_{1}$ and $r_{2}$ does not agree with the authors' experimental value at all.

Acknowledgments. The authors wish to thank Professor Mitsuru Nagasawa and Professor Zen-ichiro Kuri for their helpful discussions.

\section{REFERENCES}

1. H. Yamaoka, K. Nishiyama, K. Hayashi, and S. Okamura, Kobunshi Kagaku (Chem. High Polymers), 24, 649 (1967).

2. A. P. Sheinker, M. K. Yakovlieva, E. V. Kristalinii, and A. D. Abkin, Dokl. Acad. Nauk SSSR, 124, 632 (1959).

3. Y. Tabata, Y. Hashizume, and H. Sobue, $J$. Polym. Sci., Part A, 2, 2647 (1964).

4. Y. Tabata, Y. Hashizume, and H. Sobue, ibid., Part A, 2, 3649 (1964).

5. Y. Tsuda, ibid., 54, 193 (1961).

6. Y. Tsuda, ibid., 58, 289 (1962).

7. S. Teramachi and M. Nagasawa, J. Macromol. Sci.-Chem.; A2, 1169 (1968).

8. R. G. Fordyce, J. Amer. Chem. Soc., 69, 1903 (1947).

9. N. L. Zutty and F. J. Welch, J. Polym. Sci., 43, 445 (1960).

10. L. P. Mezhirova, A. P. Sheinker, and A. D. Abkin, Vysokomol. Soedin., 3, 99 (1961).

11. S. Teramachi and Y. Kato, J. Macromol. Sci.Chem., A4, 1985 (1970).

12. G. Goldfinger and M. Steidlitz, J. Polym. Sci., 59, 163 (1963). 